Supporting Information:

\title{
High Resolution Far-Infrared Spectroscopy of
} N-Substituted Two-Rings Polycyclic Aromatic Hydrocarbons: an extended study

\author{
S. Gruet, ${ }^{*,+, \ddagger}$ O. Pirali, ${ }^{*,+\ddagger}$ M. Goubet, ${ }^{\circledR}$ D. W. Tokaryk, ${ }^{\S}$ and P. Brechignac ${ }^{\ddagger}$ \\ $\dagger$ †ILES beamline Synchrotron SOLEIL, L'Orme des Merisiers, Saint-Aubin, France \\ $\ddagger$ Institut des Sciences Moléculaires d'Orsay (ISMO), CNRS, Univ. Paris-Sud, Université \\ Paris-Saclay, F-91405 Orsay (France) \\ ФUniv. Lille, CNRS, UMR 8523 - PhLAM - Physique des Lasers Atomes et Molécules, \\ F-59000 Lille, France \\ $\S$ Physics Department, University of New Brunswick, Fredericton, Canada \\ E-mail: sebastien.gruet@synchrotron-soleil.fr; olivier.pirali@synchrotron-soleil.fr
}


Table 1: Observed and calculated vibrational frequencies, errors ( $\delta=$ (obscalc)/obs) and harmonic intensities of quinoxaline.

n.o. $=$ not observed; $\operatorname{mix}=$ hidden below a close more intense band; M.A.E $=$ mean absolute error

\begin{tabular}{|c|c|c|c|c|c|c|}
\hline Sym. & $\begin{array}{r}\text { Obs. } \\
\left(\mathrm{cm}^{-1}\right) \\
\end{array}$ & $\begin{array}{l}\text { Harm. } \\
\left(\mathrm{cm}^{-1}\right)\end{array}$ & $\begin{array}{r}\text { Anharm. } \\
\left(\mathrm{cm}^{-1}\right)\end{array}$ & $\begin{array}{c}\delta \\
(\%) \\
\end{array}$ & $\begin{array}{c}\text { Int. } \\
\left(\mathrm{km} \cdot \mathrm{mol}^{-1}\right)\end{array}$ & Assign. \\
\hline $\mathrm{A}_{1}$ & $3040-3100$ & 3230.3 & 3099.2 & & 10.2 & $\bar{\nu} \nu_{1}$ \\
\hline $\mathrm{A}_{1}$ & $3040-3100$ & 3213.6 & 3089.8 & & 4.7 & $\nu_{2}$ \\
\hline $\mathrm{A}_{1}$ & $3040-3100$ & 3186.0 & 3040.5 & & 42.1 & $\nu_{3}$ \\
\hline $\mathrm{A}_{1}$ & 1575.3 & 1605.0 & 1566.3 & 0.6 & 2.6 & $\nu_{4}$ \\
\hline $\mathrm{A}_{1}$ & 1499.1 & 1531.7 & 1494.4 & 0.3 & 29.1 & $\nu_{5}$ \\
\hline$A_{1}$ & 1418.2 & 1447.4 & 1415.6 & 0.2 & 0.7 & $\nu_{6}$ \\
\hline$A_{1}$ & 1368.8 & 1390.7 & 1361.7 & 0.5 & 15.4 & $\nu_{7}$ \\
\hline $\mathrm{A}_{1}$ & n.o. & 1322.0 & 1296.3 & & 0.0 & $\nu_{8}$ \\
\hline $\mathrm{A}_{1}$ & 1201.1 & 1234.7 & 1203.3 & -0.2 & 1.3 & $\nu_{9}$ \\
\hline $\mathrm{A}_{1}$ & 1130.3 & 1153.6 & 1136.3 & -0.5 & 1.9 & $\nu_{10}$ \\
\hline $\mathrm{A}_{1}$ & 1022.3 & 1046.9 & 1026.9 & -0.4 & 19.8 & $\nu_{11}$ \\
\hline $\mathrm{A}_{1}$ & $\operatorname{mix}$ & 1029.9 & 1012.3 & & 1.6 & $\nu_{12}$ \\
\hline $\mathrm{A}_{1}$ & $\operatorname{mix}$ & 770.7 & 758.7 & & 4.2 & $\nu_{13}$ \\
\hline $\mathrm{A}_{1}$ & 601.5 & 610.6 & 602.7 & -0.2 & 2.9 & $\nu_{14}$ \\
\hline $\mathrm{A}_{1}$ & n.o. & 534.5 & 528.0 & & 0.0 & $\nu_{15}$ \\
\hline $\mathrm{A}_{2}$ & & 998.2 & 972.8 & & 0.0 & $\nu_{16}$ \\
\hline $\mathrm{A}_{2}$ & & 978.9 & 953.6 & & 0.0 & $\nu_{17}$ \\
\hline $\mathrm{A}_{2}$ & & 894.0 & 873.9 & & 0.0 & $\nu_{18}$ \\
\hline $\mathrm{A}_{2}$ & & 811.4 & 796.3 & & 0.0 & $\nu_{19}$ \\
\hline $\mathrm{A}_{2}$ & & 655.1 & 639.4 & & 0.0 & $\nu_{20}$ \\
\hline $\mathrm{A}_{2}$ & & 472.4 & 460.3 & & 0.0 & $\nu_{21}$ \\
\hline $\mathrm{A}_{2}$ & & 162.5 & 154.4 & & 0.0 & $\nu_{22}$ \\
\hline $\mathrm{B}_{1}$ & 961.4 & 974.8 & 952.9 & 0.9 & 3.3 & $\nu_{23}$ \\
\hline $\mathrm{B}_{1}$ & 867.2 & 871.4 & 846.2 & 2.4 & 29.9 & $\nu_{24}$ \\
\hline $\mathrm{B}_{1}$ & 756.2 & 756.7 & 737.3 & 2.5 & 53.1 & $\nu_{25}$ \\
\hline $\mathrm{B}_{1}$ & 485.3 & 491.9 & 483.8 & 0.3 & 0.1 & $\nu_{26}$ \\
\hline $\mathrm{B}_{1}$ & 400.0 & 409.4 & 402.5 & -0.6 & 10.3 & $\nu_{27}$ \\
\hline $\mathrm{B}_{1}$ & 176.9 & 175.4 & 171.9 & 2.8 & 5.7 & $\nu_{28}$ \\
\hline $\mathrm{B}_{2}$ & $3040-3100$ & 3225.5 & 3100.5 & & 8.6 & $\nu_{29}$ \\
\hline $\mathrm{B}_{2}$ & $3040-3100$ & 3201.5 & 3060.4 & & 1.8 & $\nu_{30}$ \\
\hline $\mathrm{B}_{2}$ & $3040-3100$ & 3168.1 & 3016.2 & & 4.5 & $\nu_{31}$ \\
\hline $\mathrm{B}_{2}$ & 1623.5 & 1656.3 & 1618.5 & 0.3 & 1.1 & $\nu_{32}$ \\
\hline $\mathrm{B}_{2}$ & n.o. & 1601.5 & 1553.8 & & 0.0 & $\nu_{33}$ \\
\hline $\mathrm{B}_{2}$ & $\operatorname{mix}$ & 1497.8 & 1465.1 & & 2.2 & $\nu_{34}$ \\
\hline $\mathrm{B}_{2}$ & 1386.7 & 1406.6 & 1381.2 & 0.4 & 2.3 & $\nu_{35}$ \\
\hline $\mathrm{B}_{2}$ & n.o. & 1284.2 & 1258.2 & & 0.1 & $\nu_{36}$ \\
\hline $\mathrm{B}_{2}$ & 1209.9 & 1232.7 & 1207.7 & 0.2 & 2.7 & $\nu_{37}$ \\
\hline $\mathrm{B}_{2}$ & 1100.3 & 1141.5 & 1122.6 & -2.0 & 10.2 & $\nu_{38}$ \\
\hline $\mathrm{B}_{2}$ & 953.7 & 963.1 & 949.2 & 0.5 & 19.0 & $\nu_{39}$ \\
\hline $\mathrm{B}_{2}$ & 836.4 & 847.6 & 834.9 & 0.2 & 0.9 & $\nu_{40}$ \\
\hline $\mathrm{B}_{2}$ & n.o. & 539.8 & 532.2 & & 0.2 & $\nu_{41}$ \\
\hline \multirow[t]{2}{*}{$\mathrm{B}_{2}$} & 391.7 & 394.1 & 388.3 & 0.9 & 4.5 & $\nu_{42}$ \\
\hline & & & M.A.E & 0.81 & & \\
\hline
\end{tabular}


Table 2: Observed and calculated vibrational frequencies, errors ( $\delta=$ (obscalc)/obs) and harmonic intensities of quinazoline

n.o. $=$ not observed; $\operatorname{mix}=$ hidden below a close more intense band; M.A.E $=$ mean absolute error

\begin{tabular}{|c|c|c|c|c|c|c|}
\hline Sym. & $\begin{array}{r}\text { Obs. } \\
\left(\mathrm{cm}^{-1}\right) \\
\end{array}$ & $\begin{array}{l}\text { Harm. } \\
\left(\mathrm{cm}^{-1}\right)\end{array}$ & $\begin{array}{r}\text { Anharm. } \\
\left(\mathrm{cm}^{-1}\right)\end{array}$ & $\begin{array}{c}\delta \\
(\%) \\
\end{array}$ & $\begin{array}{c}\text { Int. } \\
\left(\mathrm{km} \cdot \mathrm{mol}^{-1}\right)\end{array}$ & Assign. \\
\hline $\mathrm{A}^{\prime}$ & 3000-3100 & 3242.2 & 3113.5 & & 6.3 & $\nu_{1}$ \\
\hline $\mathrm{A}^{\prime}$ & $3000-3100$ & 3231.5 & 3105.1 & & 12.1 & $\nu_{2}$ \\
\hline $\mathrm{A}^{\prime}$ & $3000-3100$ & 3215.5 & 3090.9 & & 7.1 & $\nu_{3}$ \\
\hline $\mathrm{A}^{\prime}$ & $3000-3100$ & 3212.7 & 3079.5 & & 22.1 & $\nu_{4}$ \\
\hline $\mathrm{A}^{\prime}$ & $3000-3100$ & 3204.2 & 3065.0 & & 2.5 & $\nu_{5}$ \\
\hline $\mathrm{A}^{\prime}$ & $3000-3100$ & 3170.2 & 3040.8 & & 18.6 & $\nu_{6}$ \\
\hline $\mathrm{A}^{\prime}$ & 1630.1 & 1662.5 & 1622.0 & 0.5 & 33.4 & $\nu_{7}$ \\
\hline $\mathrm{A}^{\prime}$ & 1571.2 & 1617.2 & 1572.0 & -0.1 & 51.2 & $\nu_{8}$ \\
\hline $\mathrm{A}^{\prime}$ & 1569.1 & 1603.0 & 1568.2 & 0.1 & 49.5 & $\nu_{9}$ \\
\hline $\mathrm{A}^{\prime}$ & 1492.9 & 1524.1 & 1488.0 & 0.3 & 38.0 & $\nu_{10}$ \\
\hline $\mathrm{A}^{\prime}$ & n.o. & 1485.0 & 1459.0 & & 1.6 & $\nu_{11}$ \\
\hline $\mathrm{A}^{\prime}$ & 1410.0 & 1435.5 & 1404.7 & 0.4 & 5.3 & $\nu_{12}$ \\
\hline $\mathrm{A}^{\prime}$ & 1376.5 & 1407.4 & 1375.5 & 0.1 & 25.2 & $\nu_{13}$ \\
\hline $\mathrm{A}^{\prime}$ & 1376.5 & 1400.5 & 1369.8 & 0.5 & 14.5 & $\nu_{14}$ \\
\hline $\mathrm{A}^{\prime}$ & 1303.0 & 1340.0 & 1314.0 & -0.8 & 8.7 & $\nu_{15}$ \\
\hline $\mathrm{A}^{\prime}$ & n.o. & 1282.8 & 1259.8 & & 0.1 & $\nu_{16}$ \\
\hline $\mathrm{A}^{\prime}$ & n.o. & 1265.7 & 1246.3 & & 1.4 & $\nu_{17}$ \\
\hline $\mathrm{A}^{\prime}$ & 1207.6 & 1234.8 & 1211.7 & -0.3 & 5.5 & $\nu_{18}$ \\
\hline $\mathrm{A}^{\prime}$ & 1149.1 & 1167.4 & 1151.9 & -0.2 & 8.4 & $\nu_{19}$ \\
\hline $\mathrm{A}^{\prime}$ & 1137.8 & 1152.1 & 1133.9 & 0.3 & 9.3 & $\nu_{20}$ \\
\hline $\mathrm{A}^{\prime}$ & 1069.2 & 1103.4 & 1080.8 & -1.1 & 7.4 & $\nu_{21}$ \\
\hline $\mathrm{A}^{\prime}$ & 1011.9 & 1033.3 & 1014.9 & -0.3 & 3.2 & $\nu_{22}$ \\
\hline $\mathrm{A}^{\prime}$ & 943.8 & 953.9 & 939.2 & 0.5 & 8.7 & $\nu_{23}$ \\
\hline $\mathrm{A}^{\prime}$ & 822.7 & 834.1 & 820.9 & 0.2 & 3.4 & $\nu_{24}$ \\
\hline $\mathrm{A}^{\prime}$ & $\operatorname{mix}$ & 788.4 & 776.9 & & 3.4 & $\nu_{25}$ \\
\hline $\mathrm{A}^{\prime}$ & 634.3 & 643.0 & 636.2 & -0.3 & 11.7 & $\nu_{26}$ \\
\hline $\mathrm{A}^{\prime}$ & 531.1 & 537.8 & 531.5 & -0.1 & 0.2 & $\nu_{27}$ \\
\hline $\mathrm{A}^{\prime}$ & 513.3 & 520.5 & 514.2 & -0.2 & 1.2 & $\nu_{28}$ \\
\hline $\mathrm{A}^{\prime}$ & 372.0 & 372.3 & 369.8 & 0.6 & 1.4 & $\nu_{29}$ \\
\hline $\mathrm{A}^{\prime \prime}$ & n.o. & 996.6 & 976.3 & & 0.2 & $\nu_{30}$ \\
\hline $\mathrm{A}^{\prime \prime}$ & 985.7 & 991.8 & 972.3 & 1.4 & 0.4 & $\nu_{31}$ \\
\hline $\mathrm{A}^{\prime \prime}$ & 961.2 & 967.7 & 952.8 & 0.9 & 4.2 & $\nu_{32}$ \\
\hline $\mathrm{A}^{\prime \prime}$ & 924.2 & 929.3 & 911.4 & 1.4 & 4.6 & $\nu_{33}$ \\
\hline $\mathrm{A}^{\prime \prime}$ & 868.6 & 879.6 & 862.8 & 0.7 & 8.7 & $\nu_{34}$ \\
\hline $\mathrm{A}^{\prime \prime}$ & 793.6 & 805.5 & 792.2 & 0.2 & 11.5 & $\nu_{35}$ \\
\hline $\mathrm{A}^{\prime \prime}$ & 748.7 & 743.5 & 731.0 & 2.4 & 48.9 & $\nu_{36}$ \\
\hline $\mathrm{A}^{\prime \prime}$ & $\operatorname{mix}$ & 655.7 & 642.4 & & 4.5 & $\nu_{37}$ \\
\hline $\mathrm{A}^{\prime \prime}$ & 484.3 & 487.5 & 480.3 & 0.8 & 12.1 & $\nu_{38}$ \\
\hline $\mathrm{A}^{\prime \prime}$ & 449.6 & 452.6 & 444.6 & 1.1 & 0.0 & $\nu_{39}$ \\
\hline $\mathrm{A}^{\prime \prime}$ & 382.3 & 385.9 & 378.7 & 0.9 & 0.6 & $\nu_{40}$ \\
\hline $\mathrm{A}^{\prime \prime}$ & 174.7 & 168.1 & 164.8 & 5.7 & 0.3 & $\nu_{41}$ \\
\hline \multirow[t]{2}{*}{$A^{\prime \prime}$} & 166.3 & 161.1 & 158.1 & 4.9 & 0.1 & $\nu_{42}$ \\
\hline & & & M.A.E & 0.91 & & \\
\hline
\end{tabular}


Table 3: Observed and calculated vibrational frequencies, errors ( $\delta=$ (obscalc)/obs) and harmonic intensities of [1,5]-naphthyridine

\begin{tabular}{|c|c|c|c|c|c|c|}
\hline Sym. & $\begin{array}{r}\text { Obs. } \\
\left(\mathrm{cm}^{-1}\right)\end{array}$ & $\begin{array}{l}\text { Harm. } \\
\left(\mathrm{cm}^{-1}\right)\end{array}$ & $\begin{array}{r}\text { Anharm. } \\
\left(\mathrm{cm}^{-1}\right)\end{array}$ & $\begin{array}{c}\delta \\
(\%)\end{array}$ & $\begin{array}{c}\text { Int. } \\
\left(\mathrm{km} \cdot \mathrm{mol}^{-1}\right)\end{array}$ & Assign. \\
\hline$\overline{A_{g}}$ & & 3240.6 & 3103.3 & & 0.0 & $\nu_{1}$ \\
\hline$A_{g}$ & & 3223.2 & 3103.7 & & 0.0 & $\nu_{2}$ \\
\hline$A_{g}$ & & 3189.7 & 3048.9 & & 0.0 & $\nu_{3}$ \\
\hline$A_{g}$ & & 1645.4 & 1608.3 & & 0.0 & $\nu_{4}$ \\
\hline $\mathrm{A}_{\mathrm{g}}$ & & 1599.7 & 1561.2 & & 0.0 & $\nu_{5}$ \\
\hline$A_{g}$ & & 1503.6 & 1474.3 & & 0.0 & $\nu_{6}$ \\
\hline$A_{g}$ & & 1424.5 & 1393.6 & & 0.0 & $\nu_{7}$ \\
\hline$A_{g}$ & & 1394.3 & 1359.5 & & 0.0 & $\nu_{8}$ \\
\hline$A_{g}$ & & 1277.0 & 1250.6 & & 0.0 & $\nu_{9}$ \\
\hline$A_{g}$ & & 1144.8 & 1132.0 & & 0.0 & $\nu_{10}$ \\
\hline$A_{g}$ & & 1054.5 & 1035.4 & & 0.0 & $\nu_{11}$ \\
\hline$A_{g}$ & & 951.2 & 936.4 & & 0.0 & $\nu_{12}$ \\
\hline$A_{g}$ & & 770.6 & 757.4 & & 0.0 & $\nu_{13}$ \\
\hline $\mathrm{A}_{\mathrm{g}}$ & & 536.3 & 529.1 & & 0.0 & $\nu_{14}$ \\
\hline$A_{g}$ & & 533.8 & 527.1 & & 0.0 & $\nu_{15}$ \\
\hline$A_{u}$ & 986.4 & 997.0 & 977.8 & 0.9 & 1.7 & $\nu_{16}$ \\
\hline $\mathrm{A}_{\mathrm{u}}$ & n.o. & 949.5 & 927.6 & & 0.0 & $\nu_{17}$ \\
\hline$A_{u}$ & 817.8 & 817.7 & 800.9 & 2.0 & 78.2 & $\nu_{18}$ \\
\hline $\mathrm{A}_{\mathrm{u}}$ & 643.5 & 660.1 & 648.4 & -0.8 & 6.6 & $\nu_{19}$ \\
\hline$A_{u}$ & 479.5 & 481.5 & 475.3 & 0.9 & 1.6 & $\nu_{20}$ \\
\hline $\mathrm{A}_{\mathrm{u}}$ & 181.4 & 176.0 & 173.2 & 4.5 & 1.5 & $\nu_{21}$ \\
\hline$A_{u}$ & 166.2 & 157.4 & 153.9 & 7.4 & 7.5 & $\nu_{22}$ \\
\hline $\mathrm{B}_{\mathrm{g}}$ & & 989.2 & 969.8 & & 0.0 & $\nu_{23}$ \\
\hline $\mathrm{B}_{\mathrm{g}}$ & & 964.2 & 941.6 & & 0.0 & $\nu_{24}$ \\
\hline $\mathrm{B}_{\mathrm{g}}$ & & 847.3 & 832.6 & & 0.0 & $\nu_{25}$ \\
\hline $\mathrm{B}_{\mathrm{g}}$ & & 744.1 & 730.1 & & 0.0 & $\nu_{26}$ \\
\hline $\mathrm{B}_{\mathrm{g}}$ & & 473.9 & 463.6 & & 0.0 & $\nu_{27}$ \\
\hline $\mathrm{B}_{\mathrm{g}}$ & & 401.8 & 395.4 & & 0.0 & $\nu_{28}$ \\
\hline $\mathrm{B}_{\mathrm{u}}$ & 3087.3 & 3240.3 & 3108.8 & -0.7 & 17.5 & $\nu_{29}$ \\
\hline $\mathrm{B}_{\mathrm{u}}$ & 3087.3 & 3223.0 & 3103.0 & -0.5 & 14.2 & $\nu_{30}$ \\
\hline $\mathrm{B}_{\mathrm{u}}$ & 3032.4 & 3189.1 & 3052.6 & -0.7 & 38.2 & $\nu_{31}$ \\
\hline $\mathrm{B}_{\mathrm{u}}$ & 1599.0 & 1637.1 & 1599.8 & 0.0 & 17.5 & $\nu_{32}$ \\
\hline $\mathrm{B}_{\mathrm{u}}$ & 1494.7 & 1526.9 & 1490.4 & 0.3 & 64.5 & $\nu_{33}$ \\
\hline $\mathrm{B}_{\mathrm{u}}$ & 1396.7 & 1413.4 & 1385.4 & 0.8 & 3.8 & $\nu_{34}$ \\
\hline $\mathrm{B}_{\mathrm{u}}$ & 1305.8 & 1338.7 & 1307.9 & -0.2 & 13.3 & $\nu_{35}$ \\
\hline $\mathrm{B}_{\mathrm{u}}$ & 1211.9 & 1244.9 & 1227.2 & -1.3 & 1.9 & $\nu_{36}$ \\
\hline $\mathrm{B}_{\mathrm{u}}$ & 1190.4 & 1230.6 & 1208.9 & -1.6 & 10.5 & $\nu_{37}$ \\
\hline $\mathrm{B}_{\mathrm{u}}$ & 1101.2 & 1119.6 & 1103.2 & -0.2 & 16.9 & $\nu_{38}$ \\
\hline $\mathrm{B}_{\mathrm{u}}$ & 1016.8 & 1042.0 & 1023.6 & -0.7 & 2.7 & $\nu_{39}$ \\
\hline $\mathrm{B}_{\mathrm{u}}$ & 828.0 & 848.6 & 836.4 & -1.0 & 2.8 & $\nu_{40}$ \\
\hline $\mathrm{B}_{\mathrm{u}}$ & 601.6 & 609.9 & 602.8 & -0.2 & 14.1 & $\nu_{41}$ \\
\hline \multirow[t]{2}{*}{$\mathrm{B}_{\mathrm{u}}$} & 395.4 & 396.3 & 392.2 & 0.8 & 8.7 & $\nu_{42}$ \\
\hline & & & M.A.E & 1.28 & & \\
\hline
\end{tabular}


Table 4: Observed and calculated vibrational frequencies, errors ( $\delta=$ (obscalc)/obs) and harmonic intensities of $[1,6]$-naphthyridine

\begin{tabular}{|c|c|c|c|c|c|c|}
\hline Sym. & $\begin{array}{r}\text { Obs. } \\
\left(\mathrm{cm}^{-1}\right)\end{array}$ & $\begin{array}{l}\text { Harm. } \\
\left(\mathrm{cm}^{-1}\right)\end{array}$ & $\begin{array}{r}\text { Anharm. } \\
\left(\mathrm{cm}^{-1}\right)\end{array}$ & $\begin{array}{c}\delta \\
(\%)\end{array}$ & $\begin{array}{c}\text { Int. } \\
\left(\mathrm{km} \cdot \mathrm{mol}^{-1}\right)\end{array}$ & Assign. \\
\hline$\overline{\mathrm{A}^{\prime}}$ & "3000-3100 & 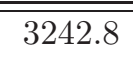 & 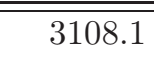 & & 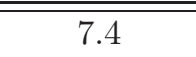 & $\overline{\nu \nu_{1}}$ \\
\hline $\mathrm{A}^{\prime}$ & $3000-3100$ & 3235.0 & 3102.1 & & 12.3 & $\nu_{2}$ \\
\hline $\mathrm{A}^{\prime}$ & $3000-3100$ & 3211.5 & 3093.4 & & 13.4 & $\nu_{3}$ \\
\hline $\mathrm{A}^{\prime}$ & $3000-3100$ & 3207.7 & 3088.0 & & 6.3 & $\nu_{4}$ \\
\hline $\mathrm{A}^{\prime}$ & $3000-3100$ & 3187.3 & 3055.8 & & 19.4 & $\nu_{5}$ \\
\hline $\mathrm{A}^{\prime}$ & $3000-3100$ & 3169.2 & 3046.1 & & 19.7 & $\nu_{6}$ \\
\hline $\mathrm{A}^{\prime}$ & 1621.0 & 1653.6 & 1613.3 & 0.5 & 76.9 & $\nu_{7}$ \\
\hline $\mathrm{A}^{\prime}$ & 1590.1 & 1627.1 & 1582.6 & 0.5 & 12.0 & $\nu_{8}$ \\
\hline $\mathrm{A}^{\prime}$ & 1554.4 & 1602.9 & 1557.7 & -0.2 & 31.8 & $\nu_{9}$ \\
\hline $\mathrm{A}^{\prime}$ & 1491.6 & 1521.1 & 1486.4 & 0.4 & 18.4 & $\nu_{10}$ \\
\hline $\mathrm{A}^{\prime}$ & 1459.4 & 1485.5 & 1455.8 & 0.3 & 6.6 & $\nu_{11}$ \\
\hline $\mathrm{A}^{\prime}$ & 1426.7 & 1448.6 & 1420.0 & 0.5 & 5.6 & $\nu_{12}$ \\
\hline $\mathrm{A}^{\prime}$ & 1372.9 & 1400.1 & 1366.1 & 0.5 & 6.9 & $\nu_{13}$ \\
\hline $\mathrm{A}^{\prime}$ & 1372.9 & 1393.1 & 1361.3 & 0.8 & 6.4 & $\nu_{14}$ \\
\hline $\mathrm{A}^{\prime}$ & 1308.3 & 1340.2 & 1313.6 & -0.4 & 9.8 & $\nu_{15}$ \\
\hline $\mathrm{A}^{\prime}$ & 1275.4 & 1292.9 & 1268.6 & 0.5 & 3.1 & $\nu_{16}$ \\
\hline $\mathrm{A}^{\prime}$ & 1239.1 & 1260.0 & 1235.1 & 0.3 & 1.8 & $\nu_{17}$ \\
\hline $\mathrm{A}^{\prime}$ & 1210.6 & 1238.2 & 1217.0 & -0.5 & 6.9 & $\nu_{18}$ \\
\hline $\mathrm{A}^{\prime}$ & 1161.4 & 1186.7 & 1164.8 & -0.3 & 1.0 & $\nu_{19}$ \\
\hline $\mathrm{A}^{\prime}$ & 1117.1 & 1140.1 & 1124.4 & -0.7 & 5.5 & $\nu_{20}$ \\
\hline $\mathrm{A}^{\prime}$ & 1029.4 & 1050.2 & 1034.0 & -0.4 & 3.2 & $\nu_{21}$ \\
\hline $\mathrm{A}^{\prime}$ & 1029.4 & 1046.4 & 1030.5 & -0.1 & 5.4 & $\nu_{22}$ \\
\hline $\mathrm{A}^{\prime}$ & 944.6 & 955.0 & 940.2 & 0.5 & 19.6 & $\nu_{23}$ \\
\hline $\mathrm{A}^{\prime}$ & 821.5 & 832.1 & 819.5 & 0.2 & 5.2 & $\nu_{24}$ \\
\hline $\mathrm{A}^{\prime}$ & 779.6 & 790.9 & 784.7 & -0.7 & 5.1 & $\nu_{25}$ \\
\hline $\mathrm{A}^{\prime}$ & 630.6 & 640.8 & 633.0 & -0.4 & 4.6 & $\nu_{26}$ \\
\hline $\mathrm{A}^{\prime}$ & 534.1 & 541.3 & 534.2 & 0.0 & 1.0 & $\nu_{27}$ \\
\hline $\mathrm{A}^{\prime}$ & 510.2 & 516.9 & 510.6 & -0.1 & 0.7 & $\nu_{28}$ \\
\hline $\mathrm{A}^{\prime}$ & 372.2 & 371.8 & 369.3 & 0.7 & 1.9 & $\nu_{29}$ \\
\hline$A^{\prime \prime}$ & 982.4 & 990.6 & 974.2 & 0.8 & 1.4 & $\nu_{30}$ \\
\hline $\mathrm{A}^{\prime \prime}$ & n.o. & 982.0 & 962.9 & & 0.0 & $\nu_{31}$ \\
\hline $\mathrm{A}^{\prime \prime}$ & n.o. & 963.9 & 945.0 & & 0.0 & $\nu_{32}$ \\
\hline$A^{\prime \prime}$ & 925.0 & 930.3 & 914.7 & 1.1 & 1.1 & $\nu_{33}$ \\
\hline$A^{\prime \prime}$ & 842.2 & 849.2 & 834.6 & 0.9 & 48.2 & $\nu_{34}$ \\
\hline $\mathrm{A}^{\prime \prime}$ & 807.9 & 821.4 & 807.8 & 0.0 & 3.1 & $\nu_{35}$ \\
\hline $\mathrm{A}^{\prime \prime}$ & 768.6 & 762.9 & 752.2 & 2.1 & 15.4 & $\nu_{36}$ \\
\hline$A^{\prime \prime}$ & 644.1 & 658.9 & 646.5 & -0.4 & 1.1 & $\nu_{37}$ \\
\hline$A^{\prime \prime}$ & 483.4 & 486.2 & 478.4 & 1.0 & 14.5 & $\nu_{38}$ \\
\hline$A^{\prime \prime}$ & 456.1 & 460.2 & 453.3 & 0.6 & 0.3 & $\nu_{39}$ \\
\hline $\mathrm{A}^{\prime \prime}$ & 379.4 & 381.8 & 374.7 & 1.2 & 2.5 & $\nu_{40}$ \\
\hline$A^{\prime \prime}$ & 177.8 & 173.6 & 171.0 & 3.8 & 1.1 & $\nu_{41}$ \\
\hline \multirow[t]{2}{*}{$\mathrm{A}^{\prime \prime}$} & 167.0 & 160.0 & 157.4 & 5.7 & 2.9 & $\nu_{42}$ \\
\hline & & & M.A.E & 0.84 & & \\
\hline
\end{tabular}


Table 5: Observed and calculated vibrational frequencies, errors ( $\delta=$ (obscalc)/obs) and harmonic intensities of indole

n.o. $=$ not observed; $\operatorname{mix}=$ hidden below a close more intense band; M.A.E $=$ mean absolute error

\begin{tabular}{|c|c|c|c|c|c|c|}
\hline Sym. & $\begin{array}{r}\text { Obs. } \\
\left(\mathrm{cm}^{-1}\right)\end{array}$ & $\begin{array}{l}\text { Harm. } \\
\left(\mathrm{cm}^{-1}\right)\end{array}$ & $\begin{array}{l}\text { Anharm. } \\
\left(\mathrm{cm}^{-1}\right)\end{array}$ & $\begin{array}{c}\delta \\
(\%)\end{array}$ & $\begin{array}{c}\text { Int. } \\
\left(\mathrm{km} \cdot \mathrm{mol}^{-1}\right)\end{array}$ & Assign. \\
\hline $\mathrm{A}^{\prime}$ & 3525.2 & 3681.9 & 3517.4 & 0.2 & 63.8 & $\nu_{1}$ \\
\hline $\mathrm{A}^{\prime}$ & $3020-3160$ & 3284.8 & 3163.1 & & 2.4 & $\nu_{2}$ \\
\hline $\mathrm{A}^{\prime}$ & $3020-3160$ & 3264.4 & 3142.4 & & 1.5 & $\nu_{3}$ \\
\hline $\mathrm{A}^{\prime}$ & $3020-3160$ & 3220.9 & 3084.8 & & 16.8 & $\nu_{4}$ \\
\hline $\mathrm{A}^{\prime}$ & $3020-3160$ & 3209.3 & 3088.2 & & 29.3 & $\nu_{5}$ \\
\hline $\mathrm{A}^{\prime}$ & $3020-3160$ & 3198.9 & 3070.5 & & 2.4 & $\nu_{6}$ \\
\hline $\mathrm{A}^{\prime}$ & $3020-3160$ & 3192.0 & 3069.2 & & 1.6 & $\nu_{7}$ \\
\hline $\mathrm{A}^{\prime}$ & 1626.2 & 1660.8 & 1616.8 & 0.6 & 2.3 & $\nu_{8}$ \\
\hline $\mathrm{A}^{\prime}$ & 1588.4 & 1619.7 & 1573.3 & 1.0 & 1.2 & $\nu_{9}$ \\
\hline $\mathrm{A}^{\prime}$ & 1519.7 & 1546.7 & 1513.3 & 0.4 & 5.5 & $\nu_{10}$ \\
\hline $\mathrm{A}^{\prime}$ & 1492.0 & 1521.9 & 1488.1 & 0.3 & 2.1 & $\nu_{11}$ \\
\hline $\mathrm{A}^{\prime}$ & 1457.7 & 1478.8 & 1445.4 & 0.8 & 23.2 & $\nu_{12}$ \\
\hline $\mathrm{A}^{\prime}$ & 1414.2 & 1443.7 & 1402.5 & 0.8 & 14.1 & $\nu_{13}$ \\
\hline $\mathrm{A}^{\prime}$ & 1347.5 & 1377.4 & 1345.8 & 0.1 & 11.1 & $\nu_{14}$ \\
\hline $\mathrm{A}^{\prime}$ & 1336.4 & 1370.5 & 1340.4 & -0.3 & 23.0 & $\nu_{15}$ \\
\hline $\mathrm{A}^{\prime}$ & 1274.2 & 1297.4 & 1270.6 & 0.3 & 14.3 & $\nu_{16}$ \\
\hline $\mathrm{A}^{\prime}$ & 1244.1 & 1263.6 & 1240.8 & 0.3 & 9.3 & $\nu_{17}$ \\
\hline $\mathrm{A}^{\prime}$ & 1204.2 & 1221.3 & 1197.0 & 0.6 & 2.6 & $\nu_{18}$ \\
\hline $\mathrm{A}^{\prime}$ & n.o. & 1164.4 & 1149.3 & & 1.0 & $\nu_{19}$ \\
\hline $\mathrm{A}^{\prime}$ & n.o. & 1136.7 & 1118.4 & & 0.1 & $\nu_{20}$ \\
\hline $\mathrm{A}^{\prime}$ & 1082.7 & 1103.9 & 1077.2 & 0.5 & 25.8 & $\nu_{21}$ \\
\hline $\mathrm{A}^{\prime}$ & 1067.3 & 1081.5 & 1063.2 & 0.4 & 4.7 & $\nu_{22}$ \\
\hline $\mathrm{A}^{\prime}$ & 1014.0 & 1031.5 & 1014.6 & -0.1 & 6.2 & $\nu_{23}$ \\
\hline $\mathrm{A}^{\prime}$ & 899.2 & 908.1 & 896.0 & 0.4 & 6.4 & $\nu_{24}$ \\
\hline $\mathrm{A}^{\prime}$ & $\operatorname{mix}$ & 884.3 & 874.1 & & 0.4 & $\nu_{25}$ \\
\hline $\mathrm{A}^{\prime}$ & 790.8 & 774.0 & 759.5 & 4.0 & 2.6 & $\nu_{26}$ \\
\hline $\mathrm{A}^{\prime}$ & 601.3 & 614.3 & 604.9 & -0.6 & 1.2 & $\nu_{27}$ \\
\hline $\mathrm{A}^{\prime}$ & n.o. & 549.4 & 542.3 & & 0.1 & $\nu_{28}$ \\
\hline $\mathrm{A}^{\prime}$ & 403.4 & 398.2 & 393.5 & 2.5 & 4.1 & $\nu_{29}$ \\
\hline $\mathrm{A}^{\prime \prime}$ & n.o. & 959.1 & 962.9 & & 0.0 & $\nu_{30}$ \\
\hline $\mathrm{A}^{\prime \prime}$ & 924.6 & 920.7 & 918.3 & 0.7 & 2.0 & $\nu_{31}$ \\
\hline$A^{\prime \prime}$ & 850.1 & 844.5 & 846.3 & 0.4 & 3.3 & $\nu_{32}$ \\
\hline $\mathrm{A}^{\prime \prime}$ & $\operatorname{mix}$ & 838.4 & 830.7 & & 0.3 & $\nu_{33}$ \\
\hline $\mathrm{A}^{\prime \prime}$ & 763.9 & 769.5 & 769.7 & -0.8 & 8.1 & $\nu_{34}$ \\
\hline$A^{\prime \prime}$ & 737.9 & 730.7 & 721.1 & 2.3 & 86.2 & $\nu_{35}$ \\
\hline$A^{\prime \prime}$ & 714.6 & 707.9 & 701.8 & 1.8 & 34.0 & $\nu_{36}$ \\
\hline $\mathrm{A}^{\prime \prime}$ & 601.3 & 611.8 & 602.8 & -0.2 & 5.4 & $\nu_{37}$ \\
\hline $\mathrm{A}^{\prime \prime}$ & 569.7 & 581.0 & 572.3 & -0.5 & 0.6 & $\nu_{38}$ \\
\hline $\mathrm{A}^{\prime \prime}$ & 419.2 & 419.4 & 415.9 & 0.8 & 5.1 & $\nu_{39}$ \\
\hline $\mathrm{A}^{\prime \prime}$ & 387.3 & 383.0 & 384.9 & 0.6 & 61.4 & $\nu_{40}$ \\
\hline $\mathrm{A}^{\prime \prime}$ & n.o. & 238.0 & 239.0 & & 0.1 & $\nu_{41}$ \\
\hline \multirow[t]{2}{*}{$\mathrm{A}^{\prime \prime}$} & 207.0 & 204.5 & 202.4 & 2.2 & 12.3 & $\nu_{42}$ \\
\hline & & & M.A.E & 0.84 & & \\
\hline
\end{tabular}


Table 6: Observed and calculated vibrational frequencies, errors ( $\delta=$ (obscalc)/obs) and harmonic intensities of quinoline

\begin{tabular}{|c|c|c|c|c|c|c|}
\hline Sym. & $\begin{array}{r}\text { Obs. } \\
\left(\mathrm{cm}^{-1}\right)\end{array}$ & $\begin{array}{l}\text { Harm. } \\
\left(\mathrm{cm}^{-1}\right)\end{array}$ & $\begin{array}{r}\text { Anharm. } \\
\left(\mathrm{cm}^{-1}\right)\end{array}$ & $\begin{array}{c}\delta \\
(\%)\end{array}$ & $\begin{array}{c}\text { Int. } \\
\left(\mathrm{km} \cdot \mathrm{mol}^{-1}\right)\end{array}$ & $\overline{\text { Assign }}$ \\
\hline$\overline{\overline{\mathrm{A}^{\prime}}}$ & "3000-3100 & ב3239.7 & 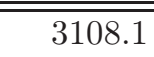 & & $\overline{90.1}$ & 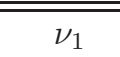 \\
\hline $\mathrm{A}^{\prime}$ & $3000-3100$ & 3231.9 & 3101.1 & & 16.2 & $\nu_{2}$ \\
\hline $\mathrm{A}^{\prime}$ & $3000-3100$ & 3228.5 & 3104.2 & & 16.4 & $\nu_{3}$ \\
\hline $\mathrm{A}^{\prime}$ & $3000-3100$ & 3213.5 & 3086.0 & & 8.9 & $\nu_{4}$ \\
\hline $\mathrm{A}^{\prime}$ & $3000-3100$ & 3204.7 & 3081.0 & & 8.9 & $\nu_{5}$ \\
\hline $\mathrm{A}^{\prime}$ & $3000-3100$ & 3200.5 & 3070.0 & & 1.6 & $\nu_{6}$ \\
\hline $\mathrm{A}^{\prime}$ & $3000-3100$ & 3184.6 & 3059.7 & & 22.1 & $\nu_{7}$ \\
\hline $\mathrm{A}^{\prime}$ & n.o. & 1662.9 & 1621.6 & & 7.6 & $\nu_{8}$ \\
\hline $\mathrm{A}^{\prime}$ & 1602.6 & 1638.8 & 1601.6 & 0.1 & 12.6 & $\nu_{9}$ \\
\hline $\mathrm{A}^{\prime}$ & 1574.7 & 1606.9 & 1568.8 & 0.4 & 12.4 & $\nu_{10}$ \\
\hline $\mathrm{A}^{\prime}$ & 1505.2 & 1539.3 & 1503.8 & 0.1 & 26.2 & $\nu_{11}$ \\
\hline $\mathrm{A}^{\prime}$ & n.o. & 1496.1 & 1468.6 & & 1.5 & $\nu_{12}$ \\
\hline $\mathrm{A}^{\prime}$ & n.o. & 1462.0 & 1431.4 & & 4.6 & $\nu_{13}$ \\
\hline $\mathrm{A}^{\prime}$ & n.o. & 1415.7 & 1386.0 & & 1.6 & $\nu_{14}$ \\
\hline $\mathrm{A}^{\prime}$ & n.o. & 1398.2 & 1366.3 & & 5.1 & $\nu_{15}$ \\
\hline $\mathrm{A}^{\prime}$ & n.o. & 1354.9 & 1325.8 & & 8.0 & $\nu_{16}$ \\
\hline $\mathrm{A}^{\prime}$ & n.o. & 1277.5 & 1255.9 & & 0.5 & $\nu_{17}$ \\
\hline $\mathrm{A}^{\prime}$ & n.o. & 1256.5 & 1228.8 & & 0.7 & $\nu_{18}$ \\
\hline $\mathrm{A}^{\prime}$ & n.o. & 1236.1 & 1214.3 & & 0.7 & $\nu_{19}$ \\
\hline $\mathrm{A}^{\prime}$ & n.o. & 1161.5 & 1144.1 & & 1.3 & $\nu_{20}$ \\
\hline $\mathrm{A}^{\prime}$ & n.o. & 1155.4 & 1138.8 & & 1.4 & $\nu_{21}$ \\
\hline $\mathrm{A}^{\prime}$ & 1118.0 & 1135.3 & 1118.0 & 0.0 & 9.8 & $\nu_{22}$ \\
\hline $\mathrm{A}^{\prime}$ & 1030.5 & 1052.1 & 1033.6 & -0.3 & 3.0 & $\nu_{23}$ \\
\hline $\mathrm{A}^{\prime}$ & 1012.7 & 1035.2 & 1018.5 & -0.6 & 3.1 & $\nu_{24}$ \\
\hline $\mathrm{A}^{\prime}$ & 939.9 & 951.3 & 934.9 & 0.5 & 4.0 & $\nu_{25}$ \\
\hline $\mathrm{A}^{\prime}$ & n.o. & 827.3 & 817.4 & & 0.8 & $\nu_{26}$ \\
\hline $\mathrm{A}^{\prime}$ & n.o. & 772.3 & 759.6 & & 1.9 & $\nu_{27}$ \\
\hline $\mathrm{A}^{\prime}$ & 611.0 & 619.5 & 612.6 & -0.3 & 6.1 & $\nu_{28}$ \\
\hline $\mathrm{A}^{\prime}$ & n.o. & 528.1 & 522.7 & & 0.2 & $\nu_{29}$ \\
\hline $\mathrm{A}^{\prime}$ & n.o. & 525.3 & 519.1 & & 0.1 & $\nu_{30}$ \\
\hline $\mathrm{A}^{\prime}$ & 375.5 & 374.7 & 371.1 & 1.2 & 4.0 & $\nu_{31}$ \\
\hline $\mathrm{A}^{\prime \prime}$ & n.o. & 989.1 & 976.0 & & 0.3 & $\nu_{32}$ \\
\hline $\mathrm{A}^{\prime \prime}$ & 977.1 & 982.2 & 972.2 & 0.5 & 1.5 & $\nu_{33}$ \\
\hline $\mathrm{A}^{\prime \prime}$ & 954.1 & 957.5 & 946.0 & 0.9 & 1.5 & $\nu_{34}$ \\
\hline $\mathrm{A}^{\prime \prime}$ & n.o. & 950.0 & 936.9 & & 0.0 & $\nu_{35}$ \\
\hline $\mathrm{A}^{\prime \prime}$ & n.o. & 875.9 & 857.9 & & 0.2 & $\nu_{36}$ \\
\hline $\mathrm{A}^{\prime \prime}$ & 803.2 & 795.0 & 782.0 & 2.6 & 58.6 & $\nu_{37}$ \\
\hline $\mathrm{A}^{\prime \prime}$ & 786.6 & 802.9 & 791.0 & -0.6 & 28.1 & $\nu_{38}$ \\
\hline $\mathrm{A}^{\prime \prime}$ & 733.0 & 721.6 & 707.7 & 3.4 & 8.0 & $\nu_{39}$ \\
\hline $\mathrm{A}^{\prime \prime}$ & n.o. & 643.4 & 635.2 & & 0.8 & $\nu_{40}$ \\
\hline $\mathrm{A}^{\prime \prime}$ & 476.3 & 476.2 & 468.7 & 1.6 & 10.0 & $\nu_{41}$ \\
\hline $\mathrm{A}^{\prime \prime}$ & n.o. & 470.1 & 460.4 & & 0.0 & $\nu_{42}$ \\
\hline $\mathrm{A}^{\prime \prime}$ & 389.5 & 394.3 & 384.9 & 1.2 & 1.7 & $\nu_{43}$ \\
\hline $\mathrm{A}^{\prime \prime}$ & 177.8 & 169.4 & 161.8 & 9.0 & 0.3 & $\nu_{44}$ \\
\hline \multirow[t]{2}{*}{$\mathrm{A}^{\prime \prime}$} & 168.4 & 159.7 & 152.2 & 9.6 & 4.7 & $\nu_{45}$ \\
\hline & & & M.A.E & 1.82 & & \\
\hline
\end{tabular}


Table 7: Observed and calculated vibrational frequencies, errors ( $\delta=$ (obscalc)/obs) and harmonic intensities of isoquinoline

\begin{tabular}{|c|c|c|c|c|c|c|}
\hline Sym. & $\begin{array}{r}\text { Obs. } \\
\left(\mathrm{cm}^{-1}\right)\end{array}$ & $\begin{array}{l}\text { Harm. } \\
\left(\mathrm{cm}^{-1}\right)\end{array}$ & $\begin{array}{r}\text { Anharm. } \\
\left(\mathrm{cm}^{-1}\right)\end{array}$ & $\begin{array}{c}\delta \\
(\%) \\
\end{array}$ & $\begin{array}{c}\text { Int. } \\
\left(\mathrm{km} \cdot \mathrm{mol}^{-1}\right)\end{array}$ & Assign \\
\hline$\overline{\mathrm{A}^{\prime}}$ & 3000-3100 & 3233.1 & 3106.3 & & $\overline{14.2}$ & $\bar{\nu} \nu_{1}$ \\
\hline $\mathrm{A}^{\prime}$ & $3000-3100$ & 3221.4 & 3081.0 & & 39.0 & $\nu_{2}$ \\
\hline $\mathrm{A}^{\prime}$ & $3000-3100$ & 3220.6 & 3090.1 & & 9.1 & $\nu_{3}$ \\
\hline $\mathrm{A}^{\prime}$ & $3000-3100$ & 3207.8 & 3086.9 & & 0.3 & $\nu_{4}$ \\
\hline $\mathrm{A}^{\prime}$ & $3000-3100$ & 3203.1 & 3074.1 & & 2.5 & $\nu_{5}$ \\
\hline $\mathrm{A}^{\prime}$ & $3000-3100$ & 3202.8 & 3099.7 & & 3.8 & $\nu_{6}$ \\
\hline $\mathrm{A}^{\prime}$ & $3000-3100$ & 3170.1 & 3051.5 & & 20.3 & $\nu_{7}$ \\
\hline $\mathrm{A}^{\prime}$ & 1634.9 & 1669.3 & 1627.8 & 0.4 & 20.7 & $\nu_{8}$ \\
\hline $\mathrm{A}^{\prime}$ & 1593.7 & 1610.2 & 1593.3 & 0.0 & 8.9 & $\nu_{9}$ \\
\hline $\mathrm{A}^{\prime}$ & 1583.9 & 1625.7 & 1580.6 & 0.2 & 17.3 & $\nu_{10}$ \\
\hline $\mathrm{A}^{\prime}$ & 1503.0 & 1535.7 & 1499.5 & 0.2 & 9.8 & $\nu_{11}$ \\
\hline $\mathrm{A}^{\prime}$ & 1462.4 & 1486.7 & 1457.0 & 0.4 & 1.1 & $\nu_{12}$ \\
\hline $\mathrm{A}^{\prime}$ & 1435.0 & 1461.9 & 1431.9 & 0.2 & 1.2 & $\nu_{13}$ \\
\hline $\mathrm{A}^{\prime}$ & 1384.1 & 1416.6 & 1382.3 & 0.1 & 3.7 & $\nu_{14}$ \\
\hline $\mathrm{A}^{\prime}$ & 1376.0 & 1398.3 & 1367.1 & 0.6 & 8.3 & $\nu_{15}$ \\
\hline $\mathrm{A}^{\prime}$ & n.o. & 1363.0 & 1340.4 & & 0.2 & $\nu_{16}$ \\
\hline $\mathrm{A}^{\prime}$ & 1271.8 & 1289.7 & 1267.4 & 0.3 & 15.2 & $\nu_{17}$ \\
\hline $\mathrm{A}^{\prime}$ & n.o. & 1271.9 & 1249.2 & & 0.3 & $\nu_{18}$ \\
\hline $\mathrm{A}^{\prime}$ & 1219.0 & 1240.0 & 1213.5 & 0.5 & 5.3 & $\nu_{19}$ \\
\hline $\mathrm{A}^{\prime}$ & n.o. & 1198.9 & 1176.9 & & 0.6 & $\nu_{20}$ \\
\hline $\mathrm{A}^{\prime}$ & 1146.8 & 1161.8 & 1145.0 & 0.2 & 1.7 & $\nu_{21}$ \\
\hline $\mathrm{A}^{\prime}$ & 1141.5 & 1153.8 & 1137.5 & 0.4 & 2.1 & $\nu_{22}$ \\
\hline $\mathrm{A}^{\prime}$ & 1036.5 & 1057.4 & 1038.8 & -0.2 & 3.0 & $\nu_{23}$ \\
\hline $\mathrm{A}^{\prime}$ & 1015.0 & 1038.2 & 1022.5 & -0.7 & 4.0 & $\nu_{24}$ \\
\hline $\mathrm{A}^{\prime}$ & 945.0 & 955.4 & 940.6 & 0.5 & 9.1 & $\nu_{25}$ \\
\hline $\mathrm{A}^{\prime}$ & 801.6 & 810.2 & 797.3 & 0.5 & 4.0 & $\nu_{26}$ \\
\hline $\mathrm{A}^{\prime}$ & n.o. & 793.9 & 787.0 & & 0.9 & $\nu_{27}$ \\
\hline $\mathrm{A}^{\prime}$ & 640.2 & 650.3 & 643.7 & -0.6 & 5.7 & $\nu_{28}$ \\
\hline $\mathrm{A}^{\prime}$ & n.o. & 529.1 & 523.3 & & 0.4 & $\nu_{29}$ \\
\hline $\mathrm{A}^{\prime}$ & 501.4 & 508.6 & 501.7 & -0.1 & 1.3 & $\nu_{30}$ \\
\hline $\mathrm{A}^{\prime}$ & n.o. & 352.9 & 350.9 & & 0.1 & $\nu_{31}$ \\
\hline $\mathrm{A}^{\prime \prime}$ & n.o. & 985.5 & 978.3 & & 0.0 & $\nu_{32}$ \\
\hline $\mathrm{A}^{\prime \prime}$ & 970.7 & 976.8 & 962.2 & 0.9 & 1.3 & $\nu_{33}$ \\
\hline $\mathrm{A}^{\prime \prime}$ & 945.0 & 956.8 & 947.6 & -0.3 & 1.8 & $\nu_{34}$ \\
\hline $\mathrm{A}^{\prime \prime}$ & n.o. & 934.7 & 919.9 & & 0.4 & $\nu_{35}$ \\
\hline $\mathrm{A}^{\prime \prime}$ & 859.2 & 862.1 & 850.5 & 1.0 & 7.7 & $\nu_{36}$ \\
\hline$A^{\prime \prime}$ & 824.9 & 825.1 & 813.9 & 1.3 & 42.4 & $\nu_{37}$ \\
\hline$A^{\prime \prime}$ & 779.3 & 791.4 & 781.1 & -0.2 & 2.4 & $\nu_{38}$ \\
\hline $\mathrm{A}^{\prime \prime}$ & 738.4 & 727.8 & 715.4 & 3.1 & 27.6 & $\nu_{39}$ \\
\hline $\mathrm{A}^{\prime \prime}$ & 636.0 & 651.4 & 642.6 & -1.0 & 4.2 & $\nu_{40}$ \\
\hline $\mathrm{A}^{\prime \prime}$ & 479.5 & 481.6 & 474.4 & 1.1 & 19.1 & $\nu_{41}$ \\
\hline $\mathrm{A}^{\prime \prime}$ & 456.7 & 459.1 & 452.3 & 1.0 & 3.4 & $\nu_{42}$ \\
\hline $\mathrm{A}^{\prime \prime}$ & 376.1 & 377.6 & 371.8 & 1.1 & 0.3 & $\nu_{43}$ \\
\hline$A^{\prime \prime}$ & 180.8 & 173.8 & 168.7 & 6.7 & 0.7 & $\nu_{44}$ \\
\hline \multirow[t]{2}{*}{$\mathrm{A}^{\prime \prime}$} & 165.6 & 158.8 & 156.5 & 5.5 & 0.3 & $\nu_{45}$ \\
\hline & & & M.А.E & 0.98 & & \\
\hline
\end{tabular}

